\title{
Modeling rhamnolipids production by Pseudomonas aeruginosa from immiscible carbon source in a batch system
}

\author{
${ }^{1}$ S. A. Medina-Moreno; ${ }^{1}$ D. Jiménez-Islas; ${ }^{1} J$. N. Gracida-Rodríguez; \\ ${ }^{2}$ M. Gutiérrez-Rojas; ${ }^{3}$ I. J. Díaz-Ramírez \\ ${ }^{1}$ Department of Biotechnological Engineering, Universidad Politécnica de Pachuca. Ex-Hacienda de Santa Bárbara, \\ Municipio, Pachuca, Hidalgo, Mexico \\ ${ }^{2}$ Department of Biotechnology, Universidad Autónoma Metropolitana Iztapalapa, Col. Vicentina, México \\ ${ }^{3}$ Academic Division of Biological Sciences, Universidad Juárez Autónoma de Tabasco, Villahermosa, \\ Tabasco, México
}

Received 30 November 2010; $\quad$ revised 20 December 2010; accepted 14 April 2011; $\quad$ available online 1 June 2011

\begin{abstract}
A mathematical model to predict the rhamnolipids production by Pseudomonas aeruginosa from oleic acid in a two phase liquid-liquid batch reaction system, was developed in this study. The model was based on two theoretical assumptions: 1) the convective oleic acid mass transfer is coupled to a bioreaction in the aqueous liquid bulk, and 2) the volume of the immiscible oleic acid drops and the saturation concentration at the interface are a function of rhamnolipids production. The model was able to accurately predict the experimental growth of the Pseudomonas aeruginosa strain, and the rhamnolipids production data with oleic acid as carbon source. This mathematical approach indicated a high correspondence between the saturation dimensionless profiles of oleic acid at the interface and the experimental profiles of surface tension difference. This modeling approach may constitute a useful tool in the design and scaling-up of bioreactors applied to the production of biosurfactants with immiscible carbon sources.
\end{abstract}

Keywords: Bioreactors; Biosurfactants; Liquid culture; Mathematical model; Oleic acid

\section{INTRODUCTION}

Biosurfactants are valuable microbial amphiphylic molecules with highly effective surface-active properties and useful in various biotechnology fields (Nitschke et al., 2005; Vyas and Dave, 2011). They are synthesized by microbial strains of different genera, especially when they grow in the presence of low water soluble substrates like petroleum hydrocarbons and waste oils (Ochsner et al., 1996; Akhavan Sepahy et al., 2005; Agarry et al., 2009). The structural diversity, low toxicity and biodegradability of biosurfactants make them ideal for use in a variety of industrial applications, i.e. cosmetic, pharmaceutical, humidifiers, preservatives and food processes as emulsifiers and recently as new players in animal and plant defense against microbes (Vatsa et al., 2010). Besides, biosurfactants have been used to improve the microbial uptake of water immiscible pollutants in bioremediation processes (Zhang and Miller, 1995; Wu et al., 2008).

\footnotetext{
\*Corresponding Author Email: idrxanum@gmail.com
} Tel.:+52993 3544 308; Fax +52993 3544308
Among the principal groups of surfactants, because of their surface activity and practical applications, the most widely studied are glycolipids mainly rhamnolipids produced by Pseudomonas aeruginosa strains. Several studies on different conditions that enhance rhamnolipids production by Pseudomonas aeruginosa have indicated that the quality and quantity of produced rhamnolipids are influenced by several factors, such as nitrogen and ion concentration, $\mathrm{pH}$, temperature and aeration rates; one of the main ones being the nature of the carbon source. Biosurfactants have been produced in aqueous systems with miscible and immiscible carbon sources (Raza et al., 2006; Abouseoud et al., 2008). Water soluble carbon sources such as glycerol, glucose and molasses (Rashedi et al., 2005) have been used to produce rhamnolipids. However, the final obtained concentration is generally one fold lower than that obtained with non-soluble substrates such as n-alkanes and vegetable oils. Mata-Sandoval et al. (2001) and Refaat (2010) compared the rhamnolipid production in 
liquid media cultures with hydrophobic and hydrophilic substrates, finding that the Pseudomonas aeruginosa UG2 strain produced around 100-165 mg of rhamnolipid/g substrate using corn oil and long chain alcohols as substrates, whereas it produced only 12$36 \mathrm{mg}$ of rhamnolipid/g substrate in the presence of hydrophylic substrates such as glucose and succinic acid. Moreover, the carbon source influences biosurfactant synthesis by either induction or catabolic repression. Chakrabarty (1985) reported that induction in the production of glycolipids by Pseudomonas aeruginosa SB30 was observed when n-alkenes were added to the culture medium, whereas catabolic repression was observed when glucose was used as a substrate. In general, when non water soluble substrates are used as a carbon source, biosynthesis may be induced to enhance biosurfactants production and obtain a better yield. This improved yield has been showed by the rhamnolipids production with Pseudomonas aeruginosa PAO1 which is able to produce high quantities of rhamnolipid during $30 \mathrm{~L}$ batch bioreactor cultivations with sunflower oil as sole carbon source and nitrogen limiting conditions ( Müller et al., 2010).

Considering the above, the production of rhamnolipids by Pseudomonas aeruginosa appears to be feasible when using low cost water immiscible substrates as carbon sources, such as vegetable and frying oils (Gautam and Tiagi, 2006; Rashedi et al., 2006). Nevertheless, the design of bioreaction systems for rhamnolipids production at micro (Rahman et al., 2010) and industrial scale (Abdel-Mawgoud et al., 2011), requires developing a model which makes it possible to describe the kinetic and mass transfer mechanisms in a heterogeneous medium. The aim of this study was to build and validate a phenomenological model to predict and analyze the production of rhamnolipids by Pseudomonas aeruginosa ATCC 9027 from oleic acid in a two phase batch reaction system. Up to now, biosurfactant production prediction has only been modeling with soluble carbon sources, being the model most recently reported by Rodrigues et al. (2006). Models that describe the uptake of microbial pollutants or product recovery in two liquid phases have been previously reported (Daugulis, 2001). However, models to describe the microbial production of biosurfactant from immiscible substrates in water, have not yet been developed, being type of great importance to design the engineering of the heterogeneous systems liquid- liquid, due to the higher yields obtained on the product (biosurfactant) with respect to the systems with soluble carbon sources. Thus, the design of the currently presented model was based on the increase of the apparent saturation solubility of oleic acid at a liquidliquid interface due to rhamnolipids produced by Pseudomonas aeruginosa. The results were compared with those obtained in a kinetic experimental assay with oleic acid as a carbon source, in order to validate the modeling data of the rhamnolipid that was produced. The model parameters were obtained either experimentally or from publications and then were fitted. The model prediction capability was tested by comparing the results with previously published data on rhamnolipids production using other immiscible substrates. This model may constitute a useful tool in the development of rhamnolipids producing bioreactors based on the use of immiscible substrates by $P$. aeruginosa. This research was carried out in the laboratory of Biotechnological Engineering of the Universidad Politécnica de Pachuca in 2009.

\section{MATERIALS AND METHODS}

Rhamnolipids production model

The model was based on the equations, conditions and variables presented in Table 1 . In general, the model considered that the convective mass transfer of the oleic acid is coupled to a bioreaction in the aqueous liquid bulk. Likewise, it was considered that the volume of the immiscible oleic acid drops and the saturation concentration at the interface are a function of the formation of rhamnolipids. Fig. 1 shows the proposed mechanisms involved in the production of rhamnolipids using immiscible substrates. The specific assumptions that supported the model were: 1) The drive force that leads to the immiscible substrate convective mass transfer at the interface is affected by the formation of the product i.e. rhamnolipids, as this increases the apparent oleic acid saturation solubility at the interface; 2) Oleic acid drops are considered to have the same size and spherical form and their volume decreases in time; 3) There are no limitations in oxygen transfer and no biomass growth on the surface of the oleic acid drops in the bioreaction system; 4) Rhamnolipids production is partly associated with biomass formation; 5) Biomass evolution time is described by the logistic equation; and 6) An unstructured sub-model was proposed to determine the dependence of the apparent oleic acid saturation solubility $\left(\mathrm{s}^{*}\right)$ of both the product 
concentration (rhamnolipid) and the critical micellar concentration (cmc). A detailed description is provided with respect to the equations that make up the model (Table 1). Eq. 1 established that oleic acid drops decrease due to the convective mass transfer from an immiscible phase to an aqueous phase. Eq. 2 described the move of the oleic acid to an aqueous phase, and its consumption by Pseudomonas aeruginosa in the same phase. Eqs. 3 and 4 are associated with a logistic model and they explain the biomass and product accumulation

Table 1: Rhamnolipids production model with definitions of parameters and variables

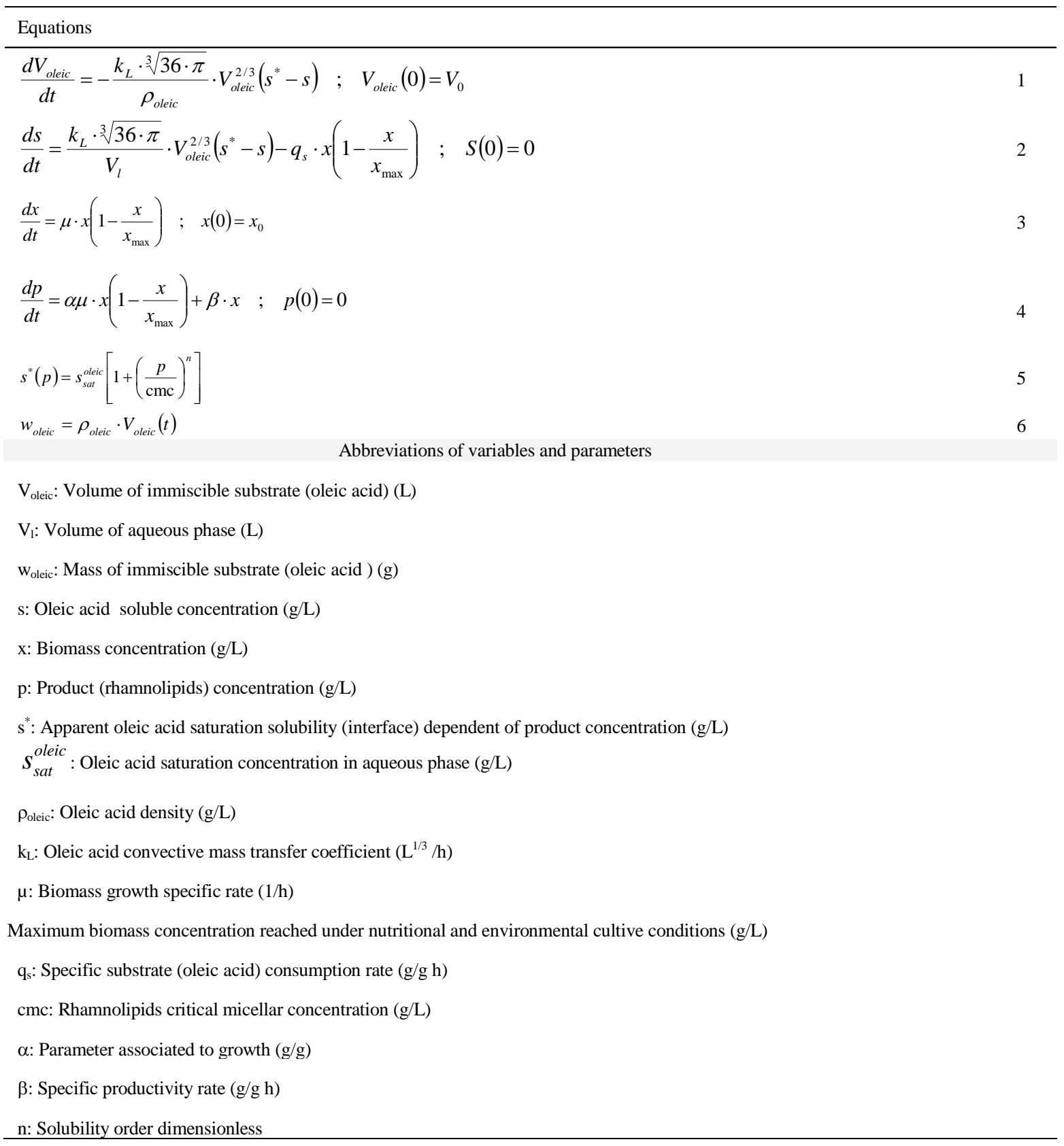


in the aqueous phase, respectively. Eq. 5 represented an unstructured relationship that describes how the apparent oleic acid saturation solubility, at the water-oleic acid interface, depends on the generation of rhamnolipids. An empirical parameter denominated solubility order (n) was introduced in this last equation. The complete deduction for the model is presented in the Rhamnolipids production model development.

\section{Microorganism}

A Pseudomonas aeruginosa strain from the ATCC collection (ATCC 9027) was kept at $4{ }^{\circ} \mathrm{C}$ on nutritive agar slants throughout the experiment. The Pseudomonas aeruginosa strain was cultured weekly in a nutritive broth and reactivated before each experimental assay.

\section{Culture medium and inoculum preparation}

Biosurfactant production assays were performed in a mineral base medium with the following composition (g/L): $\mathrm{MgSO}_{4} .7 \mathrm{H}_{2} \mathrm{O}(7), \mathrm{KCl}$ (17.5), $\mathrm{NaCl}$ (17.5), $\mathrm{CaCl}_{2}$ (0.875), $\mathrm{FeSO}_{4} .7 \mathrm{H}_{2} \mathrm{O}$ (0.0087), $\mathrm{ZnSO}_{4} .7 \mathrm{H}_{2} \mathrm{O}(0.0262), \mathrm{MnSO}_{4} .7 \mathrm{H}_{2} \mathrm{O}(0.0262), \mathrm{H}_{3} \mathrm{BO}_{3}$ (0.0052), $\mathrm{CuSO}_{4} \cdot 5 \mathrm{H}_{2} \mathrm{O}(0.002644), \mathrm{Na}_{2} \mathrm{MoO}_{4} \cdot 2 \mathrm{H}_{2} \mathrm{O}$ (0.0017) and $\mathrm{CoCl}_{2} \cdot 6 \mathrm{H}_{2} \mathrm{O}(0.002644)$. Oleic acid (Sigma, Aldrich, USA) was used as a carbon source. The nitrogen and phosphorus sources used in the experiments were sodium nitrate $\left(\mathrm{NaNO}_{3}\right)$ and phosphoric acid $\left(\mathrm{H}_{3} \mathrm{PO}_{4}\right)$, respectively. The $\mathrm{NaNO}_{3}$ and $\mathrm{H}_{3} \mathrm{PO}_{4}$ were added to obtain a carbon / nitrogen ratio $(\mathrm{C} / \mathrm{N})$ of 10 and a carbon / phosphorus ratio $(\mathrm{C} /$ $\mathrm{P}$ ) of 16 . The culture medium $\mathrm{pH}$ was adjusted to $7 \pm$ 0.2 . The density of the oleic acid was determined gravimetrically (pycnometer method) at room temperature. The propagation of the Pseudomonas aeruginosa ATCC 9027 to be used as inoculum was carried out by culturing the strain in a nutritive broth under aerobic conditions ( $\left.24 \mathrm{~h}, 30^{\circ} \mathrm{C}, 150 \mathrm{rpm}\right)$. Biomass was harvested (centrifugation at 8,100 g, $15 \mathrm{~min}$ ) and washed twice with an isotonic solution ( $\mathrm{NaCl} 0.85 \% \mathrm{w} / \mathrm{v})$. Later, the biomass suspension (mineral medium) was adjusted to 0.65 optical density units $\left(\lambda_{600 \mathrm{~nm}}\right)$.

\section{Rhamnolipids production kinetic assay}

Rhamnolipids production assays were carried out in Erlenmeyer flasks (baffled, $125 \mathrm{~mL}$ ) with $50 \mathrm{~mL}$ of sterile mineral base medium ( $\mathrm{pH}$ adjusted to $7 \pm 0.2$ ), and oleic acid ( $1 \% \mathrm{v} / \mathrm{v})$ as the only carbon source. The biomass suspension (1 mL) was used as the inoculum (Nitschke et al., 2005). Periodical sampling of the experimental units was carried out during the culture period (48 h) under constant stirring (150 rpm, $30^{\circ} \mathrm{C}$ ).

\section{Biomass growth}

The biomass was measured gravimetrically according to dry weight technique (Thaniyavarn et al., 2006). To determine along the culture the microbial growth, dry biomass $(0.2 \mu \mathrm{m}$ filtration and overnight drying $105^{\circ} \mathrm{C}$ ) was used for the construction of a standard curve, quantifying the biomass concentration by optical density $\left(\lambda_{600 \mathrm{~nm}}\right)$.

\section{Biosurfactant analysis}

Rhamnolipid production was determined following the orcinol method reported by Chandrasekaran and Bemiller (1980). It consists of an indirect measurement of the rhamnose concentration in the cell-free broth. Briefly, the cell-free broth was previously acidified to $\mathrm{pH} 2$ with $\mathrm{H}_{2} \mathrm{SO}_{4}(1 \mathrm{~N})$ and allowed to stand overnight $\left(4^{\circ} \mathrm{C}\right)$. Later, samples were centrifuged $(9,000 \mathrm{~g}$, for 10 min) and the residue was dissolved in water. Rhamnolipids were further purified by adsorption chromatography on a polystyrene resin (Sigma Aldrich, USA), as described by Reiling et al. (1986). The purified biosurfactant was obtained as an oily mixture after evaporation of the solvent in a vacuum. Rhamnolipids values were determined by multiplying the experimental data by a coefficient of 3.4, obtained from the pure rhamnolipids / rhamnose correlation reported by Benincasa et al. (2004).

\section{Surface tension measurement and critical micellar concentration}

The surface tension of all samples was determined by the ring method using a Krüs K12 tensiometer (Peker et al., 2005). All measurements were made on cell-free supernatant broth obtained after centrifugation $(8,100$ g, $25 \mathrm{~min}$ ) of the whole content of each experimental unit. The critical micellar concentration $(\mathrm{cmc})$ was calculated from the measurement of the surface tension of biosurfactant crude extract solutions. Dilutions of crude extract were prepared at different concentrations ranging from 5 to $2,000 \mathrm{mg} / \mathrm{L}$. These measurements were obtained following the ring method (Krüs K12 tensiometer). 


\section{RESULTS AND DISCUSSION}

Determination of parameters and model solution

The parameters included in the model proposed here were obtained either considering a bibliographic revision or experimentally and then were estimated by fitting the experimental data to the model obtained by simulation. Table 2 shows the model parameter values. The oleic acid saturation concentration in an aqueous phase was reported previously by Kamp et al. (2003). The oleic acid density and the cmc were determined experimentally. The second parameter was determined from the correlation between the concentration of rhamnolipids $(\mathrm{mg} / \mathrm{mL})$ and the surface tension $(\mathrm{mN} /$ $\mathrm{m}$ ). The surface tension was measured for the rhamnolipids crude extract produced by Pseudomonas aeruginosa ATCC 9027. The surface tension values drastically decreased from $\sim 72$ to $\sim 33 \mathrm{mN} / \mathrm{m}$, for the assayed rhamnolipids concentrations of 0 to $0.1 \mathrm{mg} /$ L. This surface tension reduction is comparable to the values reported by Lee et al. (2008) for a biosurfactant (a decrease from 58 to $32 \mathrm{mN} / \mathrm{m}$ ) produced by a Klebsiella sp strain isolated from waste soybean oil. Recently, Kiran et al. (2010) reported a surface tension activity of around $28 \mathrm{mN} / \mathrm{m}$ in the presence of a purified biosurfactant from the Brevibacterium aureum MSA13 strain. The values of the emulsification index and surface tension activity were greater for the biosurfactant than those obtained with synthetic surfactants. The calculation of the cmc took into account the break point of the experimental curve of concentration of rhamnolipids versus surface tension, generating a cmc value of around $0.05 \mathrm{~g} / \mathrm{L}$. The cmc value was similar to that obtained by Zhang and Miller (1995) and Raza et al. (2006). The fitted parameters given by vector $\mathrm{v}=\left(k_{L}, \mu, q_{s}, x_{\max }, \alpha, \beta, n\right)$ were obtained from the minimization of the objective function $\left(\mathrm{F}_{\mathrm{obj}}\right)$ given by (Eq. 7), coupled to the numerical solution of the dynamic model. In the function objective subindex " $\mathrm{i}$ " is the number of experimental value which has been predicted the biomass ( $\mathrm{x}$ ) and rhamnolipids (p) by the model at time $(\mathrm{t})$, and subindex $\mathrm{m}=$ total number of experimental samplings. The numerical solution of the dynamic model was obtained with a fourth order Runge-Kutta algorithm. The SIMPLEX method developed by Nelder and Mead (1965) was used to solve the optimization problem. Calculations related to the mathematical model identification and optimization was carried out using MATLAB as the software platform. To initialize the SIMPLEX optimization method, the starting values of parameters such as $\mu, q_{s}, \alpha$ and $\beta$ were taken from those reported by Raza et al. (2006) for rhamnolipids production by Pseudomonas aeruginosa growing on waste frying canola oil. The initial conditions (at $\mathrm{t}=0$ ) of both biomass and immiscible oleic acid volume were $0.1 \mathrm{~g} / \mathrm{L}$ and $0.5 \mathrm{~mL}$, respectively.

$F_{o b j}(v)=\sum_{i=1}^{m}\left(\frac{x_{i}^{\text {experimental }}-x_{i}^{\text {model }}}{x_{i}^{\text {model }}}\right)^{2}+\sum_{i=1}^{n}\left(\frac{p_{i}^{\text {experimental }}-p_{i}^{\text {model }}}{p_{i}^{\text {model }}}\right)^{2}$

Model predictions in rhamnolipids production and microbial growth

The experimental and modeling data of biosurfactant production, biomass formation and residual oleic acid during the culture of Pseudomonas aeruginosa ATCC 9027 grown on oleic acid as a carbon source are presented in Fig. 2. According to these results, the model accurately predicts the experimental proliferation of biomass and rhamnolipids production. The results observed are similar to those previously reported for different strains of Pseudomonas aeruginosa using immiscible carbon sources like waste frying olive oil (Haba et al., 2000) and linoleic acid (Thaniyavarn et al., 2006).

The decrease in oleic acid volume, the formation of biomass and the production of rhamnolipids were phenomenologically well described by the model in the following three stages (Fig. 1): 1) Initially, oleic acid transference is governed only by the difference in concentration between the interface (saturation) and the aqueous bulk phase. It presumably indicates that soluble oleic acid is beginning to be consumed by Pseudomonas aeruginosa for growth and rhamnolipids biosynthesis (Fig. 1a); 2) Later, a dynamic process is established between oleic acid mass transfer and bioreaction in the aqueous bulk phase. During this process, it is probable that the volume of the oleic acid drops decreases and the apparent oleic acid saturation concentration is modified, possibly due to interactions between the rhamnolipids and the oleic acid molecules at the liquid-liquid interface (Fig. 1b). This liquid-liquid interfacial tension is characterized by a reduction of tension at the interface, caused by the presence of biosurfactants in a two liquid phase system (Peker et al., 2005). Biosurfactants tend to absorb at the interface in an oriented arrangement, as a consequence of their amphiphatic structure that is responsible for decreasing the tension at the interface and increasing 


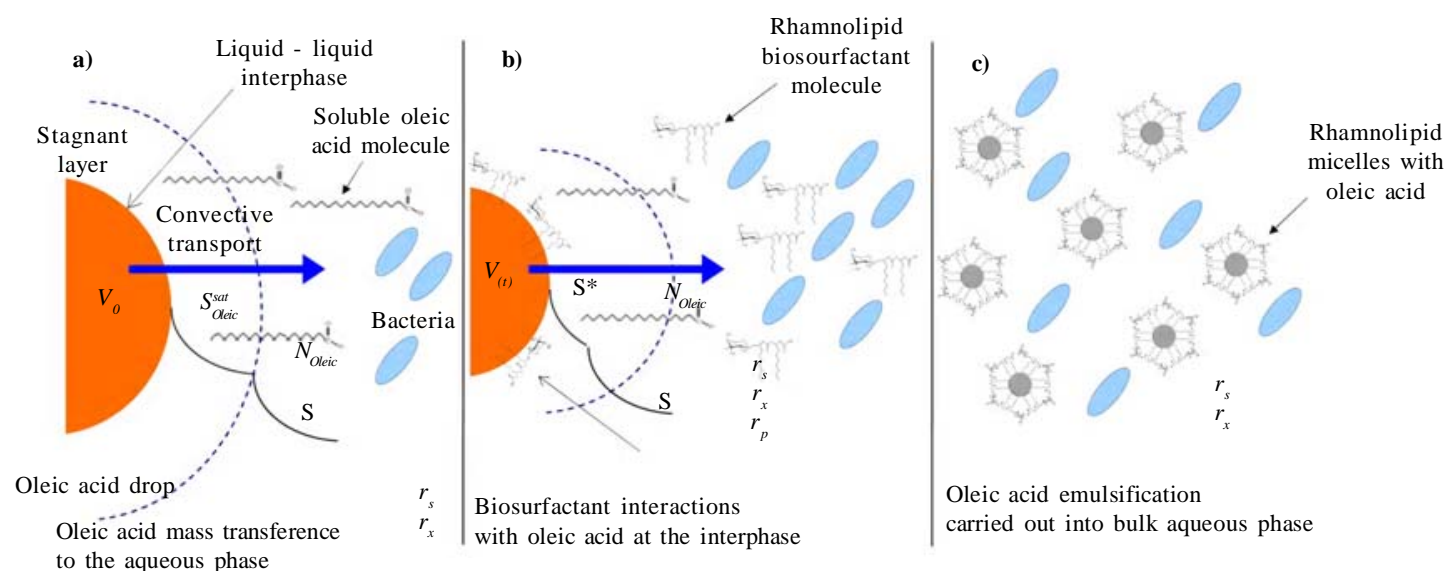

Fig. 1: Phenomenological description of rhamnolipids production by Pseudomonas aeruginosa from non-soluble oleic acid in a two liquid phase batch system. a) Initial stage where the oleic acid mass transference and the aqueous phase bioreaction occur; b) A dynamic process is established between oleic acid mass transference, bioreaction in the aqueous bulk phase and rhamnolipids interaction at the interface; c) The oleic acid is emulsified

Table 2: Parameter values used for the development of the proposed model

\begin{tabular}{|c|c|c|c|}
\hline Parameter & Initial values & Fitted values & Unities \\
\hline Oleic acid saturation concentration in aqueous phase $\left(s_{\text {sat }}^{\text {oleic }}\right)^{*}$ & - & 0.0017 & $\mathrm{~g} / \mathrm{L}$ \\
\hline Oleic acid density $\left(\rho_{\text {oleic }}\right) * *$ & - & 890 & $\mathrm{~g} / \mathrm{L}$ \\
\hline Rhamnolipids critical micellar concentration (cmc) & - & 0.05 & $\mathrm{~g} / \mathrm{L}$ \\
\hline Oleic acid convective mass transfer coefficient $\left(\mathrm{k}_{\mathrm{L}}\right)$ & 50 & 90.25 & $\mathrm{~L}^{1 / 3} / \mathrm{h}$ \\
\hline Specific substrate (oleic acid) consumption rate $\left(\mathrm{q}_{\mathrm{s}}\right)$ & 1.25 & 0.483 & $\mathrm{~g} /(\mathrm{g} \mathrm{h})$ \\
\hline Biomass growth specific rate $(\mu)$ & 0.31 & 0.174 & $1 / \mathrm{h}$ \\
\hline $\begin{array}{l}\text { Maximum biomass concentration reached under nutritional and environmental } \\
\text { cultive conditions }\left(\mathrm{x}_{\max }\right)\end{array}$ & 1.60 & 1.556 & $\mathrm{~g} / \mathrm{L}$ \\
\hline Parameter associated to growth $(\alpha)$ & 1.85 & 0.064 & g/g \\
\hline Specific productivity rate $(\beta)$ & 0.57 & 0.0017 & $\mathrm{~g} /(\mathrm{g} \mathrm{h})$ \\
\hline Solubility order (n) & 1.00 & 0.125 & dimensionless \\
\hline
\end{tabular}

* Kamp et al. (2003); **Experimental: Raza et al. (2006)

the miscibility between non-miscible phases. 2) In the last stage, the critical micellar concentration of the rhamnolipids is reached, a stable emulsion is produced and, consequently, the maximum apparent solubility for the oleic acid is achieved. Then, the convective mass transfer is a non-predominant process. Moreover, biomass formation and oleic acid consumption occur from the emulsified oleic acid (Fig. 1c). In order to demonstrate the predictive capacity of the model, a simulation was carried out using two groups of previously reported experimental data. Results are presented in Fig. 3. Graphics were constructed from both the data reported and data generated by the model. The simulation was used to describe the production of rhamnolipids and biomass by two different strains: 1 ) Pseudomonas aeruginosa 47T2 (Haba et al., 2000) and 2) Pseudomonas aeruginosa A41 (Thaniyavarn et al., 2006), growing on waste frying olive oil and linoleic 


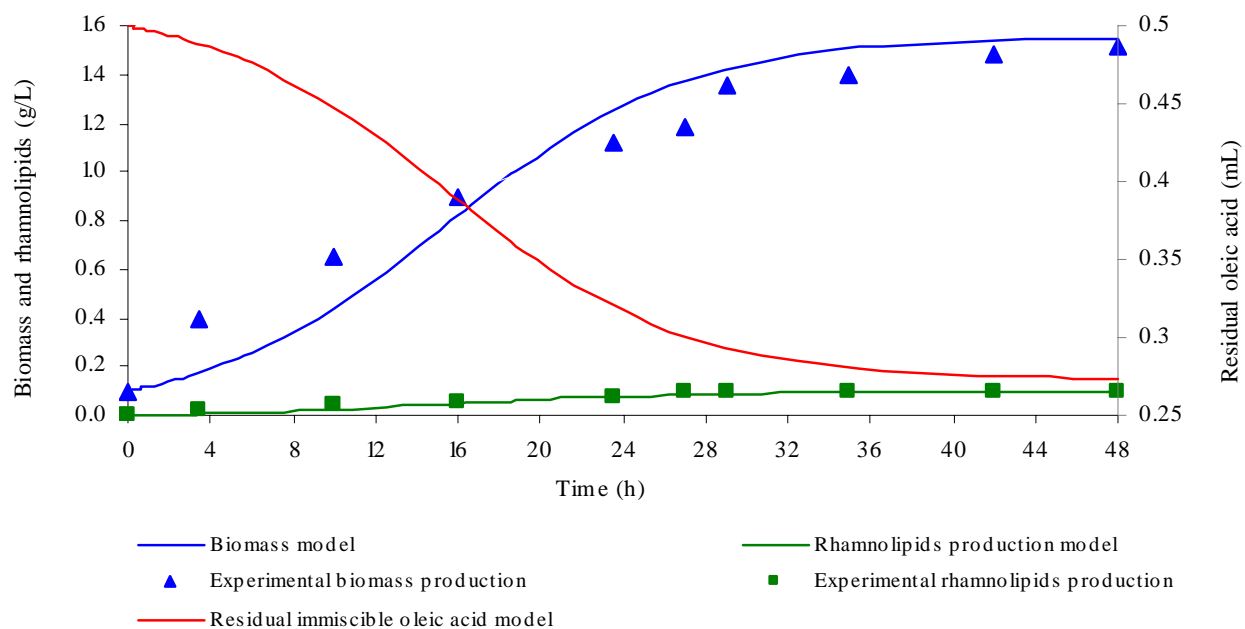

Fig. 2: Model prediction for rhamnolipids production and Pseudomonas aeruginosa ATCC 9027 biomass progress from immiscible oleic acid

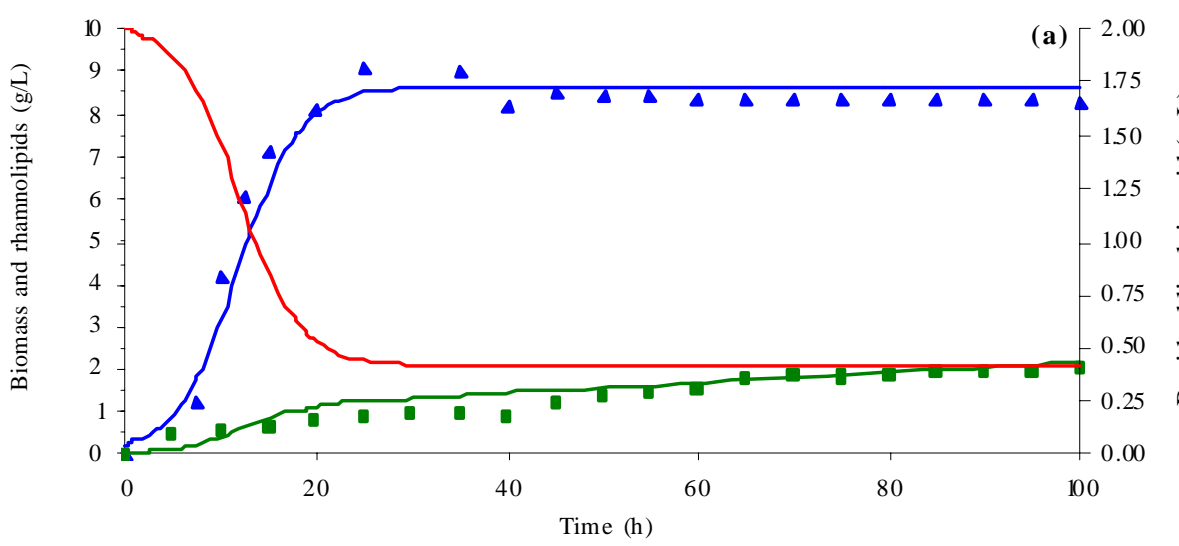

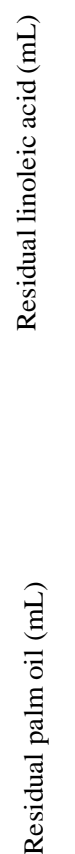
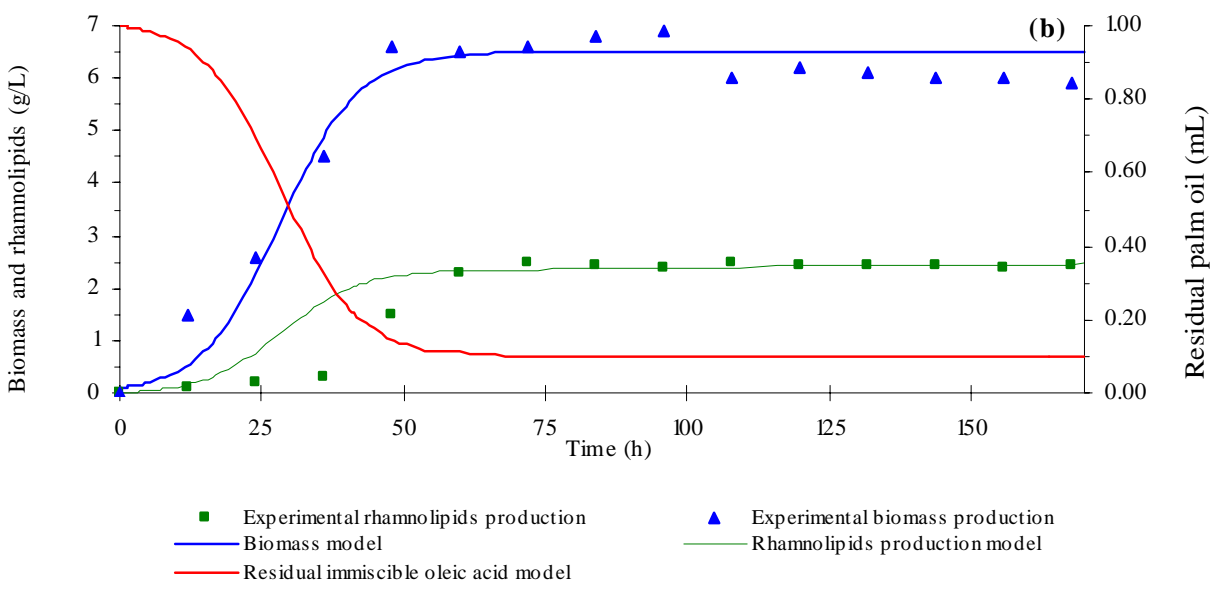

Fig. 3: Model predictions for the Pseudomonas aeruginosa strains: a) 47T2 on waste frying olive oil (Haba et al., 2000) and; b) A41 on linoleic acid (Thaniyavarn et al., 2006). Graphics were constructed from both the data reported and data generated by the model 
acid, respectively. For both simulations, the values of cmc, density, saturation concentration and convective mass transfer coefficient were the same as those used for the oleic acid simulation. The other values $\left(\mu, q_{s}\right.$, $\left.x_{\max }, \alpha, \beta, n\right)$ were estimated by fitting the experimental data previously reported with the SIMPLEX optimization method (Table 3). The aqueous phase volume used in both cases was the same as that used in the present study ( $50 \mathrm{~mL})$. In the case of rhamnolipids production, the parameters recorded for the waste frying olive oil were $0.2 \mathrm{~g} / \mathrm{L}$ for the initial value of the biomass and $2 \mathrm{~mL}$ for the immiscible phase volume, while those recorded for linoleic acid were $0.1 \mathrm{~g} / \mathrm{L}$ and $1 \mathrm{~mL}$, respectively. The results obtained with the model proposed here indicated that both groups of experimental data were described with accuracy and consistency by the model. Results were comparable to those obtained using oleic acid experimental data for the simulation. This analysis shows that the model can successfully predict rhamnolipids production and microbial growth of Pseudomonas aeruginosa strains in a two phase (liquid-liquid) batch system. The model may be considered a useful tool for the design and scaling-up of bioreactors for the production of biosurfactants. Nevertheless, at a large scale it is necessary to consider the common relationship between stirring and foam formation, particularly when the use of antifoam in this type of system is not recommended and also because rhamnolipids had an effect on the apparent solubility and mass transfer of the immiscible carbon sources. Studies under abiotic conditions should be conducted to independently ascertain the values of the above mentioned parameters at concentrations below the critical micellar concentration.

\section{Predictions of oleic acid mass transfer and solubilization}

The apparent solubility of oleic acid at the interface may be modified by interactions with rhamnolipids during the first two stages of the process (Fig. 1). Consequently, the interfacial tension decreases with the formation of the biosurfactant amphiphatic structure, which also modifies the mass transfer of immiscible substrates. This global effect is well described by the proposed unstructured Eq. 5 that is a key part of the model. According to the above mentioned, the apparent solubility of oleic acid at the interface $\left(\mathrm{s}^{*}\right)$ is strongly linked to the surface tension (s). Fig. 4 presents the predictions of the model for the dimensionlessed apparent solubility $\left(\mathrm{S}_{\mathrm{dim}}=\mathrm{s}^{*} / \mathrm{s}^{*}{ }_{\max }\right)$, the oleic acid soluble concentration in the aqueous phase (s), and the experimental dimensionlessed surface tension difference $\left(\Delta \sigma_{\text {dim }}=\left[\sigma_{0}-\sigma(\mathrm{t})\right] / \Delta \sigma_{\max }\right.$, where $\sigma_{0}$ corresponds to the surface tension at $\mathrm{t}=0$ ). The model was able to predict a high correspondence between the temporal profiles of the $S_{\text {dim }}$ and the experimental $\Delta \sigma_{\text {dim }}$. According to the profiles, the apparent oleic acid solubility increases in parallel with the surface tension, achieving a maximum approximately after $16 \mathrm{~h}$ of culture. At this time, the rhamnolipids concentration was 52 $\mathrm{mg} / \mathrm{L}$ and the $\mathrm{cmc}$ was recorded around this value. Regarding the aqueous phase bulk solubility (s), the model generated a profile with a fast decrease for oleic

Table 3: Parameters used to validate the proposed model. Values of rhamnolipids production were obtained from previously reported results using two different strain of Pseudomonas aeruginosa growing on waste frying olive oil (Haba et al., 2000) and linoleic acid (Thaniyavarn et al., 2006)

\begin{tabular}{|c|c|c|c|}
\hline Parameter & $\begin{array}{c}\text { Olive oil frying } \\
\text { wastes }\end{array}$ & Linoleic acid & Unities \\
\hline Specific substrate (oleic acid) consumption rate $\left(\mathrm{q}_{\mathrm{s}}\right)$ & 0.705 & 0.408 & $\mathrm{~g} /(\mathrm{g} \mathrm{h})$ \\
\hline Biomass growth specific rate $(\mu)$ & 0.315 & 0.147 & $\mathrm{~L} / \mathrm{h}$ \\
\hline Maximum biomass concentration reached under nutritional and & 8.66 & 6.57 & $\mathrm{~g} / \mathrm{L}$ \\
\hline \multicolumn{4}{|l|}{ environmental cultive conditions $\left(\mathrm{x}_{\max }\right)$} \\
\hline Parameter associated to growth $(\alpha)$ & 0.13 & 0.36 & $\mathrm{~g} / \mathrm{g}$ \\
\hline Specific productivity rate $(\beta)$ & 0.015 & 0.0016 & $\mathrm{~g} /(\mathrm{g} h)$ \\
\hline Solubility order (n) & 0.205 & 0.315 & dimensionless \\
\hline
\end{tabular}




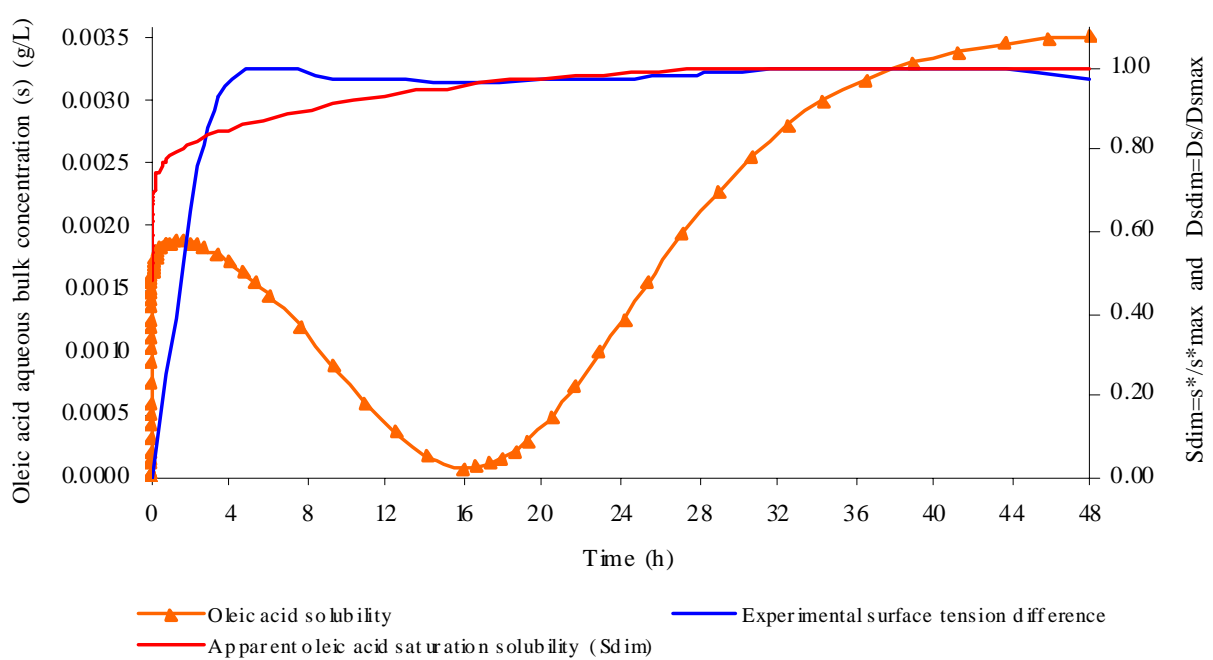

Fig. 4: Model predictions for dimensionless apparent oleic acid saturation solubility at the interface $\left(\mathrm{S}_{\mathrm{dim}}=\mathrm{s}^{*} / \mathrm{s}_{\text {max }}^{*}\right.$, oleic acid solubility in the aqueous phase (s), and the dimensionlessed experimental surface tension difference $\left(\Delta \sigma_{\mathrm{dim}}\right)$

acid, once it was available in the aqueous phase (4-16 $\mathrm{h}$, time range in which exponential growth occurs). Later, the s increased until it reached a value similar to the maximum of the apparent saturation solubility (3.5 $\mathrm{mg} / \mathrm{L})$ at the interface. Apart from allowing predictions on the behavior of the biomass and rhamnolipids, the model also made it possible to describe both the mass transfer and the change in the apparent saturation solubility at the interface using oleic acid as a carbon source.

\section{Rhamnolipids production model development}

Eq. 8 represents the basic mass balance of the immiscible oleic acid. It establishes that the uptake rate responds to the flux through the interface from the liquid-liquid to the aqueous phase:

$$
\frac{d w_{\text {oleic }}}{d t}=-A_{\text {oleic }} \cdot N_{\text {oleic }}
$$

In this equation, $\mathrm{w}_{\text {oleic }}$ is the immiscible oleic acid mass, $A_{\text {oleic }}$ is the superficial mass transfer surface of the oleic acid drops, and $\mathrm{N}_{\text {oleic }}$ is the oleic acid convective mass flux. The convective mass flux in the process is due to the oleic acid concentration difference between the apparent saturation solubility at the interface $\left(\mathrm{s}^{*}\right)$ and the aqueous phase bulk solubility (s). Besides, the flux depends on the resistance to mass transfer due to the mixing regime, which is characterized by a convective mass transfer coefficient $\left(\mathrm{k}_{\mathrm{L}}\right)$. Therefore the flux of oleic acid is given by:

$N_{\text {oleic }}=k_{L}\left(s^{*}-s\right)$

Assuming also that the oleic acid drops are spherical, the mass transfer surface and drop volumes can be related by the radius of a sphere $\left[r=\left(A_{\text {oleic }} / 4 \pi\right)^{1 / 2}=\left(3 \cdot V_{\text {oleic }} / 4 \pi\right)^{1 / 3}\right.$ ] where:

$A_{\text {oleic }}=\sqrt[3]{36 \cdot \pi} \cdot V_{\text {oleic }}^{2 / 3}$

Moreover, taking into account that $w_{\text {oleic }}$ depends both on the volume and the density of the oleic acid drops (here it is considered that $\rho_{\text {oleic }}$ remains constant), and substituting Eqs. 9 and 10 in the mass balance Eq. 8, Eq. 1 of the model (Table 1) is obtained.

Regarding the soluble oleic acid, the mass balance establishes that its accumulation in the aqueous phase is equal to the inlet rate minus the uptake rate (due to consumption by Pseudomonas aeruginosa) 
in the same phase, as is described by:

$$
\frac{d w_{\text {oleic }}^{\text {soluble }}}{d t}=A_{\text {oleic }} \cdot N_{\text {oleic }}-\frac{r_{x}}{Y_{x / s}} \cdot V_{l}
$$

In this equation, $r_{x}$ is equal to the biomass growth rate, $\mathrm{Y}_{\mathrm{x} / \mathrm{s}}$ indicates the biomass-substrate yield, and $\mathrm{V}_{1}$ represents the aqueous phase volume. The terms $\mathrm{A}_{\text {oleic }}$ and $\mathrm{N}_{\text {oleic }}$ for the convective flux transport, are the same as those shown in Eqs. 9 and 10.

In order to calculate $r_{x}$, the logistic equation was used, instead of the Monod model, to describe the biomass growth. This was considered as growth not limited by the uptake of a soluble substrate, but by the transport and solubilization rates. The growth rate described through the logistic equation is given by Eq. 3 of the model (Table 1).

Writing $w_{\text {oleic }}^{\text {solub }}$ in terms of the volume of the aqueous phase and the soluble oleic acid concentration (s), and substituting Eqs. 9, 10 and 3 in Eq. 11, generates Eq. 2 of the model ( Table 1) to describe the evolution of the soluble oleic acid concentration. In this equation, the specific consumption rate $\left(\mathrm{q}_{\mathrm{s}}\right)$ is obtained from the ratio of the specific biomass growth rate $(\mu)$ and the biomasssubstrate yield $\left(\mathrm{Y}_{\mathrm{x} / \mathrm{s}}\right)$.

The rhamnolipids mass balance (Eq. 12) pecifies that accumulation is equal to production rate, as a result of the synthesis from oleic acid carried out by Pseudomonas aeruginosa.

Regarding the production rate, the Leudeking and Piret model was used to describe this parameter, as it is versatile for fitting product formation data obtained from several fermentation processes (Bailey and Ollis, 1986).

$$
\frac{d w_{\text {rhamnol }}}{d t}=r_{p} \cdot V_{l}=\left(\alpha \cdot r_{x}+\beta \cdot x\right) \cdot V_{l}
$$

Writing the rhamnolipids mass $\left(w_{\text {rhamnol }}\right)$ in terms of aqueous phase volume and the rhamnolipids concentration (p) and substituting Eq. 3 in Eq. 11, Eq. 4 of the model (Table 1) is obtained to describe the evolution of rhamnolipids concentration.

Furthermore, the main point during the development of the model was taking into account the effect of rhamnolipids concentration on the oleic acid saturation solubility at the interface. In the course of rhamnolipids biosynthesis, the amphiphylic and micellar interactions between biosurfactants and immiscible oleic acid, allow two events to take place simultaneously: 1) an increase in the apparent saturation solubility; and 2) a decrease in the size of the drops. A non-structured model was purposed to describe this behavior (Eq. 5; Table 1).

This equation involves two physicochemical parameters (oleic acid saturation concentration and micellar concentration) and introduces one empirical parameter, denominated here as solubility order (n). Therefore, Eqs. 1 to 5 describe: 1) the transport; 2) the kinetic processes; and c) the equilibrium processes that are involved in the biosynthesis of rhamnolipids by Pseudomonas aeruginosa from an immiscible substrate such as oleic acid in a batch system.

\section{CONCLUSION}

The mathematical model was capable of accurately predicting $P$. aeruginosa ATCC 9027 growth and production of rhamnolipids using oleic acid as the only carbon source. Previously reported rhamnolipids production data were described successfully by this model. Moreover, the model exhibits a high correlation between the dimensionless profiles of oleic acid saturation and the experimental profiles of surface tension difference.

The validation strategy indicated that the developed modeling-approach may be a valuable tool to design and scale-up bioreactors for the production of biosurfactants. Further studies including the establishment of methods that make it possible to determine relevant parameters of mass transport and interface interactions in heterogeneous mixedsystems should be addressed.

\section{ACKNOWLEDGEMENTS}

The authors wish to thank the Mexican National Science and Technology Council (CONACyT) for financial support (Project \#. 54910).

\section{REFERENCES}

Abdel-Mawgoud, A. M.; Hausmann, R.; Lépine, F.; Müller, M. M.; Déziel, E., (2011). Rhamnolipids: detection, analysis, biosynthesis, genetic regulation and bioengineering of production. In: Soberón-Chávez(Ed), Biosurfactants from genes to applications, Microbiology monographs series, Springer-Verlag, Berlin Heidelberg. 
Abouseoud, M.; Maachi, R.; Amrane, A.; Boudergua, S.; Nabi, A., (2008). Evaluation of different carbon and nitrogen sources in production of biosurfactant by Pseudomonas fluorescens., Desalination., 223 (1-3), 143-151 (9 pages).

Agarry, S. E.; Audu, T. O. K.; Solomon, B. O., (2009). Substrate inhibition kinetics of phenol degradation by Pseudomonas fluorescence from steady state and washout data. Int. J. Environ. Sci. Tech., 6 (3), 443-450 (8 pages).

Akhavan Sepahy, A.; Mazaheri Assadi, M.; Saggadian , V.; Noohi, A., (2005). Production of biosurfactant from Iranian oil fields by isolated Bacilli. Int. J. Environ. Sci. Tech., 1 (4), 287-293 (7 pages).

Bailey, J. E.; Ollis, D. F., (1986). Biochemical engineering fundamentals., McGraw-Hill International.

Benincasa, M.; Abalos, A.; Oliveira, I.; Manresa, A., (2004). Chemical structure, surface properties and biological activities of the biosurfactant produced by Pseudomonas aeruginosa LBI from soapstock., Anton. Van Lee., 85 (1), 1-8 (8 pages).

Chakrabarty, A. M., (1985). Genetically manipulated microorganisms and their products in the oil service industries. Trends Biotech., 3 (2), 32-38 (7 pages).

Chandrasekaran, E. V.; Bemiller, J. N., (1980). Constituent analyses of glycosaminoglycans. Whistler, R. L., Wolfrom, M. L. (Eds.), Methods in Carbohydrate Chemistry, Academic Press, NY.

Daugulis, A. J., (2001). Two-phase partitioning bioreactors: a new technology platform for destroying xenobiotics. Trends. Biotech., 19 (11), 457-462 (6 pages).

Gautam, K. K.; Tiagi, V. K., (2006). Microbial surfactants: A review. J. Oleo Sci., 55 (4), 155-166 (12 pages).

Haba, E.; Espuny, M. J.; Busquets, M.; Manresa, A., (2000). Screening and production of rhamnolipids by Pseudomonas aeruginosa 47T2 NCIB from waste frying oils. J. Appl. Microbiol., 88 (3), 379-387 (9 pages).

Kamp, F.; Guo, W.; Souto, R.; Pilch, P. F.; Corkey, B. E.; Hamilton, J. A., (2003). Rapid flip-flop of oleic acid across the plasma membrane of adipocytes. J. Biol. Chem., 278 (10), 7988-7995 (8 pages).

Kiran, G. S.; Thomas, T. A.; Selvin, J.; Sabarathnam, B.; Lipton, A.P., (2010). Optimization and characterization of a new lipopeptide biosurfactant produced by marine Brevibacterium aureum MSA13 in solid state culture. Bioresour. Tech., 101 (7), 2389-2396 (8 pages).

Lee, S. C.; Lee, S. J.; Kim, S. H.; Park, I. H.; Lee, Y. S., (2008). Characterization of new biosurfactant produced by Klebsiella sp. Y6-1 isolated from waste soybean oil. Bioresour. Tech., 99 (7), 2288-2292 (5 pages).

Mata-Sandoval, J. C.; Karns, J.; Torrents, A., (2001). Effect of nutritional and environmental conditions on the production and composition of rhamnolipids by $P$. aeruginosa UG2. Microbiol. Res., 155 (4), 249-256 (8 pages).

Müller, M. M.; Hormann, B.; Syldatk, C.; Hausmann, R., (2010). Pseudomonas aeruginosa PAO1 as a model for rhamnolipid production in bioreactor system. Appl. Microbiol. Biotech., 87 (1), 167-174 (8 pages).
Nelder, J. A.; Mead, R., (1965). A simple method for function minimization. Comput. J., 7 (4), 308-313 (6 pages).

Nitschke, M.; Costa, S. G.; Contiero, J., (2005). Rhamnolipid surfactants: an update on the general aspects of these remarkable biomolecules. Biotech. Prog., 21 (6), 1593-1600 (8 pages).

Ochsner, U.; Hembach, T.; Fiechter, A., (1996). Production of rhamnolipids biosurfactants. Adv. Biochem. Engin. Biotech., 53, 89-118 (30 pages).

Peker, S.; Helvaci, S.; Özdemir, G., (2005). Interfacesubphase interactions of rhamnolipids in aqueous rhamnose solutions. Langmuir, 19 (14), 5838-5845 (8 pages).

Rahman, P. K. S. M.; Pasiray, G.; Auger, V.; Ali, Z., (2010). Production of rhamnolipid biosurfactants by Pseudomonas aeruginosa DS10-129 in a microfluidic bioreactor. Biotech. Appl. Biochem., 55 (1), 45-52 (8 pages).

Rashedi, H.; Mazaheri Assadi, M.; Bonakdarpour, B.; Jamshidi, E., (2005). Environmental importance of rhamnolipids production from molasses as a carbon source. Int. J. Environ. Sci. Tech., 2 (1), 59-62 (4 pages).

Rashedi, H.; Mazaheri Assadi, M.; Jamshidi, E.; Bonakdarpour, B., (2006). Production of rhamnolipids by Pseudomonas aeruginosa growing on carbon sources. Int. J. Environ. Sci. Tech., 3 (3), 297-303 (7 pages).

Raza, Z. A.; Khan, M. S.; Khalid, Z. M.; Rehman, A., (2006). Production kinetics and tensoactive characteristics of biosurfactant from Pseudomonas aeruginosa mutant grown on waste frying oils. Biotech. Lett., 20, 1623-1631 (9 pages).

Refaat, A. A., (2010). Different techniques for the production of biodiesel from waste vegetable oil. Int. J. Environ. Sci. Tech., 7 (1), 183-213 (31 pages).

Reiling, H. E.; Thanci-Wyss, U.; Guerra-Santos, L. H.; Hirt, R.; Kappeli, O.; Fiechter, A., (1986). A pilot plan production of rhamnolipid biosurfactant by Pseudomonas aeruginosa. Appl. Environ. Microbiol., 51 (5), 985-989 (5 pages).

Rodrigues, L.; Moldes, A.; Teixeira, J.; Oliveira, R., (2006). Kinetic study of fermentative biosurfactant production by Lactobacillus strains. Biochem. Eng. J., 28 (2), 109116 (8 pages).

Thaniyavarn, J.; Chongchin, A.; Wanitsuksombut, N.; Thaniyavarn, S.; Pinphanichakarn, P.; Leepipatpiboon, N.; Morikawa, M.; Kanaya, S., (2006). Biosurfactant production by Pseudomonas aeruginosa A41 using palm oil as carbon source. J. Gen. Appl. Microbiol., 52 (4), 215-222 (8 pages).

Vatsa, P.; Sanchez, L.; Clement, C.; Baillieu, F.; Dorey, S., (2010). Rhamnolipid biosurfactants as new players in animal and plant defense against microbes. Int. J. Mol. Sci., 11 (12), 5095-5108 (16 pages).

Vyas, T. K.; Dave, B. P., (2011). Production of biosurfactant by Nocardia otitidiscaviarum and its role in biodegradation of crude oil. Int. J. Environ. Sci. Tech., 8 (2), 425-432 (8 pages). 
Modeling rhamnolipids production by Pseudomonas aeruginosa

Wu, J. Y.; Yeh, K. L.; Lu, W. B.; Lin, C. B.; Chang, J. S., (2008).

Rhamnolipids production with indigenous Pseudomonas aeruginosa EM1 isolated from oil-contaminated site.

Bioresour. Tech., 99 (5), 1157-1164 (8 pages).
Zhang, Y.; Miller, R. M., (1995). Enhanced octadecane dispersion and biodegradation by a Pseudomonas rhamnolipid surfactant (biosurfactant), Appl. Environ. Microbiol., 58(10), 3276-3282 (7 pages).

\section{AUTHOR (S) BIOSKETCHES}

Medina-Moreno, S. A., Ph.D., Department of Biotechnological Engineering, Universidad Politécnica de Pachuca. Ex-Hacienda de Santa Bárbara, Municipio, Pachuca, Hidalgo, Mexico. Email: samm67@upp.edu.mx

Jiménez-Islas, D., Ph.D., Department of Biotechnological Engineering, Universidad Politécnica de Pachuca. Ex-Hacienda de Santa Bárbara, Municipio, Pachuca, Hidalgo, Mexico. Email: donaislas@yahoo.com.mx

Gracida-Rodríguez, J. N., Ph.D., Department of Biotechnological Engineering, Universidad Politécnica de Pachuca. Ex-Hacienda de Santa Bárbara, Municipio, Pachuca, Hidalgo, Mexico. Email: gracidaj@netscape.net

Gutiérrez-Rojas, M., Department of Biotechnology, Universidad Autónoma Metropolitana Iztapalapa, Pachuca, Hidalgo, Col. Vicentina, México D.F. Email: mgr@xanum.uam.mx

Díaz-Ramírez, I. J., Ph.D., Academic Division of Biological Sciences, Universidad Juárez Autónoma de Tabasco, Villahermosa, Tabasco, México. Email: idrxanum@gmail.com

How to cite this article: (Harvard style)

Medina-Moreno, S. A.; Jiménez-Islas, D.; Gracida-Rodríguez, J. N.; Gutiérrez-Rojas, M.; Díaz-Ramírez, I. J., (2011). Modeling rhamnolipids production by Pseudomonas aeruginosa from immiscible carbon source in a batch system. Int. J. Environ. Sci. Tech., 8 (3), 471-482. 